Article

\title{
Ishige okamurae Ameliorates Methylglyoxal-Induced Nephrotoxicity via Reducing Oxidative Stress, RAGE Protein Expression, and Modulating MAPK, Nrf2/ARE Signaling Pathway in Mouse Glomerular Mesangial Cells
}

\author{
Mingyeong Kim ${ }^{1,+(\mathbb{D})}$, Chiheung Cho ${ }^{2,+} \mathbb{D}$, Changjun Lee ${ }^{2,3}$, Bomi Ryu $^{4} \mathbb{D}$, Sera Kim ${ }^{2} \mathbb{D}$, Jinyoung Hur ${ }^{2}$ \\ and Sang-Hoon Lee $1,2, *$ (D) \\ 1 Department of Food Biotechnology, University of Science and Technology, Daejeon 34113, Korea; \\ 50034@kfri.re.kr \\ 2 Division of Functional Food Research, Korea Food Research Institute, 245 Nongsaengmyeong-ro, Iseo-myeon, \\ Wanju-gun 55365, Korea; chiheungcho@kfri.re.kr (C.C.); 50040@kfri.re.kr (C.L.); k.sera@kfri.re.kr (S.K.); \\ jyhur@kfri.re.kr (J.H.) \\ 3 Department of Food Science and Technology, Chonbuk National University, Jeonju City 54896, Korea \\ 4 Marine Science Institute, Jeju National University, Jeju 63333, Korea; ryu.bomi@gmail.com \\ * Correspondence: shnlee@kfri.re.kr; Tel.: +82-063-219-9357; Fax: +82-063-219-9876 \\ + Both authors contributed equally to this work.
}

Citation: Kim, M.; Cho, C.; Lee, C. Ryu, B.; Kim, S.; Hur, J.; Lee, S.-H. Ishige okamurae Ameliorates Methylglyoxal-Induced Nephrotoxicity via Reducing Oxidative Stress, RAGE Protein Expression, and Modulating MAPK, Nrf2/ARE Signaling Pathway in Mouse Glomerular Mesangial Cells. Foods 2021, 10, 2000. https:/ / doi.org/10.3390/foods 10092000

Academic Editor: Oliver Chen

Received: 27 July 2021

Accepted: 24 August 2021

Published: 26 August 2021

Publisher's Note: MDPI stays neutral with regard to jurisdictional claims in published maps and institutional affiliations.

Copyright: (c) 2021 by the authors. Licensee MDPI, Basel, Switzerland. This article is an open access article distributed under the terms and conditions of the Creative Commons Attribution (CC BY) license (https:/ / creativecommons.org/licenses/by/ $4.0 /)$.

\begin{abstract}
Advanced glycation end-products (AGEs) such as methylglyoxal (MGO) play a vital role in the pathogenesis of nephropathy, a diabetic complication. In the present study, we evaluated the anti-glycation and renal protective properties of Ishige okamurae extract (IOE) against AGE-induced oxidative stress. HPLC analysis confirmed that bioactive phlorotannins such as diphlorethohydroxycarmalol and ishophloroglucin A are predominantly present in IOE. IOE showed strong anti-glycation activities via inhibition of AGE formation, inhibition of AGE-protein cross-linking, and breaking of AGE-protein cross-links. In addition, in vitro studies using mesangial cells demonstrated that IOE effectively suppressed intracellular reactive oxygen species production, intracellular MGO accumulation, and apoptotic cell death by MGO-induced oxidative stress, in addition to regulating the expression of proteins involved in the receptor for AGEs and nuclear factor erythroid 2-related factor 2 (Nrf2)/antioxidant response elements (ARE) signaling pathways. Therefore, IOE can serve as a natural therapeutic agent for the management of AGE-related nephropathy.
\end{abstract}

Keywords: Ishige okamurae; advanced glycation end-product; nephropathy; methylglyoxal; Nrf2/ARE pathway

\section{Introduction}

Advanced glycation end-products (AGEs) can be generated via a glycation reaction (known as the Maillard reaction) between the carbonyl group of a reducing sugar (glucose, fructose, or ribose) in blood and the free amino group of a protein [1]. In the early stage, the condensation of glucose and amines produces an unstable Schiff base. The Schiff base is then arranged to form Amadori products. During the degradation of Amadori products, reactive carbonyl species are formed in the intermediate stage. At the advanced stage, a cross-link occurs between dicarbonyl intermediates and proteins (e.g., collagen), resulting in irreversible glycation end-products [2]. These adducts accelerate the development and progression of diabetic complications by altering the normal physiological functions of a protein upon glycation [3]. In addition, AGEs induce reactive oxygen species (ROS; e.g., superoxide $\left(\mathrm{O}_{2}^{-}\right)$, hydrogen peroxide $\left(\mathrm{H}_{2} \mathrm{O}_{2}\right)$, peroxynitrite $\left.\left(\mathrm{ONOO}^{-}\right)\right)$, which cause intracellular oxidative stress and promote the mitochondrial apoptosis pathway by interacting with the receptor for AGEs (RAGE) [4]. The increase in AGE formation in chronic hyperglycemic conditions is a major contributor on the pathogenesis of diabetic complications 
including retinopathy, neuropathy, and nephropathy [5]. Therefore, inhibition of AGE formation or accumulation in the body is extensively regarded as the most effective ways for preventing or delaying AGE-related diabetic complications.

In hyperglycemic patients, there is an increase in the levels of methylglyoxal (MGO), which is one of the reactive glycation end-products and the major intermediate and precursor for AGE formation [6]. MGO is more reactive than glucose, with a stronger ability to cross-link with the arginine or lysine residues of proteins, leading to the formation of stable end-products called AGEs and subsequent activation of RAGE, which then initiates various diabetic complications [6,7]. However, MGO can directly impair cellular function independently of the AGE-RAGE pathway through intracellular reactive oxygen species (ROS) generation, inducing oxidative damage to proteins, apoptosis by inducing oxidative stress, and increasing caspase activity [5]. Several synthetic compounds, such as aminoguanidine (AG) and the thiazolium-derived compound ALT-711 (alagebrium) have been shown to inhibit the cross-linking of proteins and slow down the progression of diabetes-related complications. However, these agents were ultimately not approved for clinical use due to safety and efficacy issues during the clinical trial phase [8,9]. Therefore, the scavenging of AGEs may be a promising therapeutic approach for diabetes and its associated complications, and there is a need for the development of effective AGE-scavenging agents that are based on natural resources.

Ishige okamurae (I. okamurae) is a brown seaweed that is distributed throughout the temperate coastal region, and is generally enrich in the coast of Jeju Island, Korea. I. okamurae contains various natural antioxidant substances, among which phlorotannins are the most powerful water-soluble antioxidants $[10,11]$. Several previous studies have reported that I. okamurae has a variety of biological benefits associated with phlorotannins, including anti-inflammatory, anti-hypertensive, anti-bacterial, anti-tumor, anti-obesity, and free radical-scavenging activities [12,13]. I. okamurae acts as an inhibitor of alpha-glucosidase, which is a key enzyme in the modulation of glucose absorption, and thus enhances glucose homeostasis [14]. In addition, a phlorotannin-rich extract from I. okamurae has been shown to have a positive effect on genetically diabetic mice. Among the many bioactive compounds isolated from I. okamurae, phlorotannins have been extensively studied for their health benefits. In particular, diphlorethohydroxycarmalol (DPHC) has been considered to influence responses relevant to diabetes through the modulation of glucose-induced oxidative stress, as well as the inhibition of carbohydrate-digestive enzymes [15]. Although many previous studies have been conducted on the various biological benefits of I. okamurae, its preventive effects on AGE-related diabetic complications have not been investigated so far.

Brown seaweeds, which are rich in phlorotannins, are consumed for the prevention or improvement of chronic metabolic diseases due to their beneficial biological properties. On the basis of these investigations, we supposed that the phlorotannins contained in I. okamurae may have protective effects against AGE-induced renal damage. Therefore, in the present study, we evaluated the inhibitory effects of I. okamurae on AGE formation and AGE cross-links, as well as its nephroprotective effects against AGE-induced oxidative stress in mouse glomerular mesangial cells. In addition, the underlying mechanisms of action were partly elucidated using a protein expression analysis of key signaling pathways such as RAGE, MAPKs, and Nrf2/ARE.

\section{Materials and Methods}

\subsection{Chemicals}

Aminoguanidine (AG), glucose, fructose, bovine serum albumin (BSA), sodium azide, 3,3,5,5-tetramethylbenzidine (TMB) liquid substrate system for enzyme-linked immunosorbent assay (ELISA), MGO, sodium phosphate monobasic, and sodium phosphate dibasic were purchased from Sigma (St. Louis, MO, USA). Dulbecco's modified Eagle's medium/F12 Nutrient Mixture (DMEM/F-12; Ham, 3:1 mixture) was obtained from Welgene Inc. (Daegu, Korea). Fetal bovine serum (FBS), hydroxyethyl-piperazineethane-sulfonic acid 
(HEPES), penicillin/streptomycin solution, and collagen I-coated plates were purchased from Gibco (Rockville, MD, USA). PRO-PREP ${ }^{\mathrm{TM}}$ Protein Extraction Solution, the OxiSelect ${ }^{\mathrm{TM}}$ Methylglyoxal (MG) Competitive ELISA Kit, and the RAGE antibody were purchased from iNtRON Biotechnology (Seongnam, Korea), Cell Biolabs Inc. (San Diego, CA, USA), and Merck Millipore (Billerica, MA, USA), respectively. Antibodies against catalase (CAT), heme oxygenase 1 (HO-1), NAD(P)H: quinone oxidoreductase 1 (NQO1), extracellular signal-regulated kinases (ERK), phospho-ERK (p-ERK), c-Jun N-terminal kinase (JNK), phosphor-JNK (p-JNK), p38, phosphor-p38 (p-p38), and superoxide dismutase 1 (SOD1) were purchased from Cell Signaling Technology (Danvers, MA, USA). $\beta$-actin and nuclear factor erythroid 2-related factor (Nrf2) antibodies were obtained from Santa Cruz Biotechnology (Santa Cruz, CA, USA). The receptor for advanced glycation end-products (RAGE) antibody was obtained from Merck Millipore (Billerica, MA, USA).

\subsection{Sample Preparation}

In brief, Ishige okamurae (I. okamurae) was collected from Jeju Island, South Korea. I. okamurae was washed with tap water to remove salt, sand, and epiphytes attached to the surface. Next, I. okamurae was lyophilized and ground to obtain a dry powder. Dried I. okamura powder was extracted in 50\% ethanol under reflux conditions. The extract was concentrated and freeze-dried (final yield: $87 \mathrm{~g}$ ). I. okamurae extracts (IOEs; kindly provided by Prof. Y.J. Jeon) were sealed and stored at $-20^{\circ} \mathrm{C}$ prior to use.

\subsection{Identification of Phlorotannins in I. okamurae Using HPLC}

Chromatographic analyses were conducted on an Acquity ${ }^{\mathrm{TM}}$ Arc equipped with a 2998 PDA detector and an Acquity ${ }^{\mathrm{TM}}$ QDa detector system (Waters Corporation, Beverly, MA, USA). The phlorotannin compounds in I. okamurae extract were analyzed using a Poroshell 120 EC-C18 column $(4 \mu \mathrm{m}, 4.6 \mathrm{~mm} \times 100 \mathrm{~mm}$; Agilent(Santa Clara, CA, USA)). The mobile phase consisted of (A) $0.1 \%$ formic acid in water and (B) acetonitrile containing $0.1 \%$ formic acid. The HPLC elution condition consisted of an isocratic system of $32 \% \mathrm{~B}$ at a flow rate of $0.4 \mathrm{~mL} / \mathrm{min}$ and an injected volume of $10 \mu \mathrm{L}$.

\subsection{Inhibitory Effect of I. okamurae on AGE Formation}

The inhibiting ability of IOE on AGE formation via the Maillard reaction was evaluated according to the method described by Do et al. [16], with slight modifications. BSA $(10 \mathrm{mg} / \mathrm{mL})$, glucose $(100 \mathrm{mM})$ and fructose $(100 \mathrm{mM})$ were incubated in phosphatebuffered saline (PBS, pH 7.4 with $0.02 \%$ sodium azide), in the presence or absence of AG $(0.5 \mathrm{mM})$ or IOE $(5,20$, and $100 \mu \mathrm{g} / \mathrm{mL})$ at $37^{\circ} \mathrm{C}$ for 7 day. AGE formation level was measured at an excitation wavelength of $350 \mathrm{~nm}$ and an emission wavelength of $450 \mathrm{~nm}$, using a microplate reader (Molecular Devices, Sunnyvale, CA, USA). The AGE formation level was expressed as a percentage (\%) decrease in the fluorescence intensity of the control (without test samples).

\subsection{AGE Cross-Link Breaking Effect of I. okamurae}

The effect of IOE on breaking AGE-BSA-induced cross-linking with collagen was evaluated according to the method of Kim et al. [17] with slight modifications. To confirm the IOE-induced breaking activity of AGE-BSA-induced cross-linking of collagen, $1 \mathrm{mg} / \mathrm{mL}$ of AGE-BSA was added to collagen-coated 96-well plates and incubated at $37^{\circ} \mathrm{C}$ for $4 \mathrm{~h}$, following which the AGE-BSA complexes with collagen were incubated in either the presence or absence of ALT-711 or IOE at $37^{\circ} \mathrm{C}$ for $18 \mathrm{~h}$. The unattached AGE-BSA was washed with $0.05 \%$ PBST. Breaking levels were detected using TMB substrate. Breaking of AGE-induced cross-links was expressed in terms of percentage decrease in optical density. 


\subsection{AGE Cross-Link Inhibitory Effect of I. okamurae}

The inhibitory effect of IOE on AGE-BSA-induced cross-linking to collagen was evaluated according to the method described by Do et al. [18], with slight modifications. To confirm the inhibitory effect of IOE on AGE-induced cross-linking to collagen, $1 \mathrm{mg} / \mathrm{mL}$ of AGE-BSA was incubated with AG $(0.5 \mathrm{mM})$ or $\operatorname{IOE}(5,20$, and $100 \mu \mathrm{g} / \mathrm{mL})$ on collagencoated 96-well plates at $37^{\circ} \mathrm{C}$ for $18 \mathrm{~h}$. The inhibitory ability of IOE was detected using TMB as a substrate. Inhibition of AGE-induced cross-linking was expressed in terms of percentage decrease in optical density.

\subsection{Cell Culture}

Mouse glomerular mesangial cells were cultured in a 3:1 mixture of DMEM/F-12 medium containing $5 \% \mathrm{FBS}, 14 \mathrm{mM}$ HEPES, $100 \mathrm{U} / \mathrm{mL}$ penicillin, and $100 \mu \mathrm{g} / \mathrm{mL}$ streptomycin at $37{ }^{\circ} \mathrm{C}$ in $5 \% \mathrm{CO}_{2}$ and $95 \%$ air.

\subsection{Nephroprotective Effect of I. okamurae}

The nephroprotective effects of IOE were determined using a 3-(4,5-dimethylthiazol-2yl)-2,5-diphenyl tetrazolium bromide (MTT) reduction assay. Mesangial cells were seeded at a density of $3.0 \times 10^{4}$ cells per well in a 96-well plate with $100 \mu \mathrm{L}$ of DMEM/F12 medium containing 5\% FBS and incubated for $6 \mathrm{~h}$ before sample treatment. The supernatant was removed and the cells were pretreated with various concentrations of IOE for $1 \mathrm{~h}$, followed by treatment with MGO for $24 \mathrm{~h}$. After removing the medium with samples, $0.5 \mathrm{mg} / \mathrm{mL}$ MTT reagent was added to the cells and incubated for $3 \mathrm{~h}$ at $37^{\circ} \mathrm{C}$. The resulting formazan product was dissolved in dimethyl sulfoxide. The number of viable cells was determined by measuring the absorbance at $570 \mathrm{~nm}$ (detection wavelength) and $630 \mathrm{~nm}$ (reference wavelength) using a microplate reader (Infinite M200; Tecan Austria GmbH, Grödig, Austria). Viability was expressed in terms of percentage (\%) of absorbance of the control cells (without MGO and IOE).

\subsection{Intracellular ROS Scavenging Activity of I. okamurae}

The intracellular antioxidant capacity was determined using a fluorescent probe $\left(2^{\prime}, 7^{\prime}\right.$ dichlorofluorescin diacetate or DCFH-DA). Mouse glomerular mesangial cells were seeded at a density of $3.0 \times 10^{4}$ cells per well in a 96-well plate with $100 \mu \mathrm{L}$ of DMEM/F12 medium containing 5\% FBS and incubated for $6 \mathrm{~h}$. The supernatant was subsequently removed, following which the cells were treated with various concentrations of IOE for $1 \mathrm{~h}$, and then treated with MGO for $2 \mathrm{~h}$. The cells were then washed twice with Hank's balanced salt solution (HBSS) and incubated with $10 \mu \mathrm{M}$ DCFH-DA in HBSS for $30 \mathrm{~min}$. Fluorescence was measured using an Infinite M200 microplate reader. ROS production levels in the cells were measured at excitation and emission wavelengths of 485 and $530 \mathrm{~nm}$, respectively. The degree of intracellular ROS levels was expressed as percentage (\%) of the non-treated group (without MGO and IOE).

\subsection{MGO-Derived AGE Concentration}

The ability of IOE to inhibit intracellular AGE accumulation was evaluated by measuring the concentrations of MGO-cross-linked proteins using the OxiSelect ${ }^{\mathrm{TM}}$ Methylglyoxal (MG) Competitive ELISA Kit (Cell biolabs, San Diego, CA, USA). Mesangial cells were seeded at a density of $1.0 \times 10^{6}$ cells per 6-well plate and treated with MGO, with or without IOE, for $24 \mathrm{~h}$. The cells were then collected and lysed to measure the MGO-derived AGE concentrations using an ELISA kit, by measuring the absorbance at $450 \mathrm{~nm}$ using an Infinite M200 microplate reader.

\subsection{Apoptosis Analysis}

Induction of apoptosis was measured using the Muse ${ }^{\mathrm{TM}}$ Annexin V \& Dead Cell Kit (Luminex, Austin, TX, USA). Mesangial cells were seeded at a density of $1.0 \times 10^{6}$ cells per 6-well plate with $2 \mathrm{~mL}$ of DMEM/F12 medium containing 5\% FBS and incubated for $6 \mathrm{~h}$. 
Cells were pretreated with various concentrations of IOE for $1 \mathrm{~h}$. Following that, MGO was added to the wells to induce apoptosis for $23 \mathrm{~h}$. After removing the medium with samples and MGO, the cells were washed with cold HBSS and diluted to a density of 500 cells $/ \mu \mathrm{L}$ prior to staining. Cells were resuspended in DMEM/F12 medium, added to the Muse ${ }^{\mathrm{TM}}$ Annexin V \& Dead Cell Reagent, and then incubated for $20 \mathrm{~min}$ at room temperature in the dark. The assay results were measured using a Muse ${ }^{\mathrm{TM}}$ Cell Analyzer (Merck Millipore, Sydney, Australia). Results have been expressed in terms of percentage of total apoptotic cells (early and late apoptotic cells), percentage of live cells, and percentage of dead cells.

\subsection{Western Blotting}

The pretreated cells were scraped, and intracellular proteins were extracted using PRO-PREP ${ }^{\text {TM }}$ Protein Extraction Solution for Cell/Tissue containing 1\% protease and phosphatase (Thermo Fisher Scientific, Rockford, IL, USA). First, the protein contents were measured using a DC Protein Assay (Bio-Rad, Hercules, CA, USA), following which the protein content of each group was diluted to the same concentration. After being bathed in a metal bath at $100{ }^{\circ} \mathrm{C}$ for $5 \mathrm{~min}$, the protein was cooled to room temperature and used as a backup. The remaining protein samples were stored at $-80^{\circ} \mathrm{C}$ until use. The diluted protein samples were subjected to electrophoresis at $200 \mathrm{~V}$ using Any kD ${ }^{\mathrm{TM}}$ Mini-PROTEAN ${ }^{\circledR}$ TGX Stain-Free ${ }^{\mathrm{TM}}$ Gels, following which the separated proteins were transferred to the Trans-Blot ${ }^{\circledR}$ Turbo $^{\mathrm{TM}}$ RTA Transfer Kit polyvinylidene difluoride membrane (Bio-Rad, Hercules, CA, USA). The membranes were blocked with 5\% skim milk in Tris-buffered saline with $0.1 \%$ Tween-20 for $1 \mathrm{~h}$. The membranes were then incubated with primary antibodies (used at a dilution of 1:1000) with weak shaking overnight at $4{ }^{\circ} \mathrm{C}$. After incubation, the membranes were incubated with HRP-labeled goat anti-rabbit IgG or goat anti-mouse $\operatorname{IgG}$ (used at a dilution of 1:5000) for $1 \mathrm{~h}$ at room temperature. Finally, the membrane was exposed to WesternSure ${ }^{\circledR}$ Chemiluminescent Substrate (LI-COR, Lincoln, NE, USA), and luminescence was detected using ChemiDoc ${ }^{\mathrm{TM}}$ (Bio-Rad, Hercules, CA, USA).

\subsection{Statistical Analysis}

All experiments were independently performed in triplicate, and data are presented as mean \pm standard deviation $(n=3)$. To assess the significant differences in the mean values, one-way analysis of variance was performed followed by the Tukey's multiple comparisons test $(p<0.05)$ using GraphPad Prism ver. 9 (GraphPad Software, Inc., San Diego, CA, USA).

\section{Results}

\subsection{Identification of Phlorotannins in I. okamurae}

Marine algae produce metabolites necessary for the growth and propagation of the organism involved with developmental stages and further defense responses against external environmental changes. Enabling the nutraceutical use of I. okamurae requires the identification and quantification of its chemical components and biological activity. DPHC and IPA were identified as the predominant bimodal peaks in the chromatogram obtained for I. okamurae (Figure 1). The mass spectrum of each phlorotannin was determined systemically by comparison with previous studies on the identification of I. okamurae composition $[19,20]$; which is phlorotannins in the form of organic polymers of phloroglucinol (1,3,5-trihydroxybenzene), DPHC (512.06 g/mol, $7.1 \mathrm{~min})$, and IPA (1986.26 g/mol, $22.2 \mathrm{~min}$ ). The concentrations of DPHC and IPA in the I. okamurae used in this study were $2.61 \pm 0.27 \%$ and $2.43 \pm 0.44 \%$, respectively. 


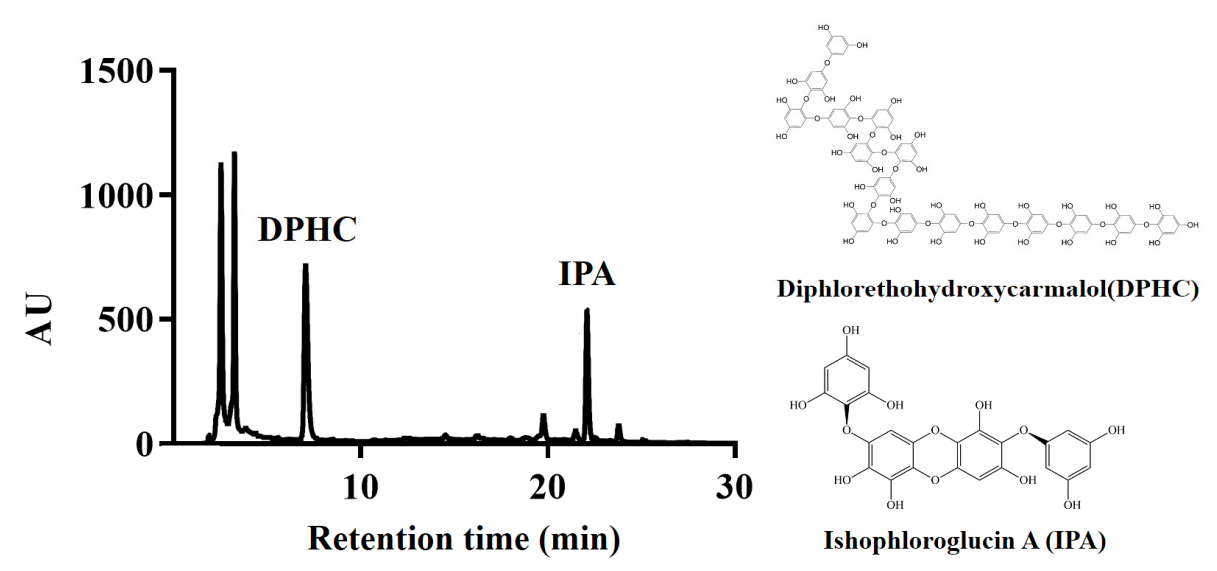

Figure 1. HPLC chromatogram of Ishige okamurae extract. It was confirmed that Ishige okamurae $50 \%$ ethanol $(v / v)$ extract contained two phlorotannin compounds diphlorethohydroxycarmalol (DPHC) and ishophloroglucin A (IPA), which were identified.

\subsection{Anti-Glycation Abilities of I. okamurae}

In our study, we evaluated the in vitro anti-glycation property of IOE (Figure 2). An AGE formation assay was performed to assess the inhibitory ability of IOE on AGE formation. As shown in Figure 2A, incubation with BSA-glucose and -fructose induced AGE formation. However, incubation with $\operatorname{IOE}(5,20$, and $100 \mu \mathrm{g} / \mathrm{mL})$ significantly $(* * * p 0.001)$ reduced AGE formation. In addition, the inhibitory effect of IOE on AGE formation was similar to that of $0.5 \mathrm{mM} \mathrm{AG}$, a synthetic compound developed to prevent diabetes complications.

(A)

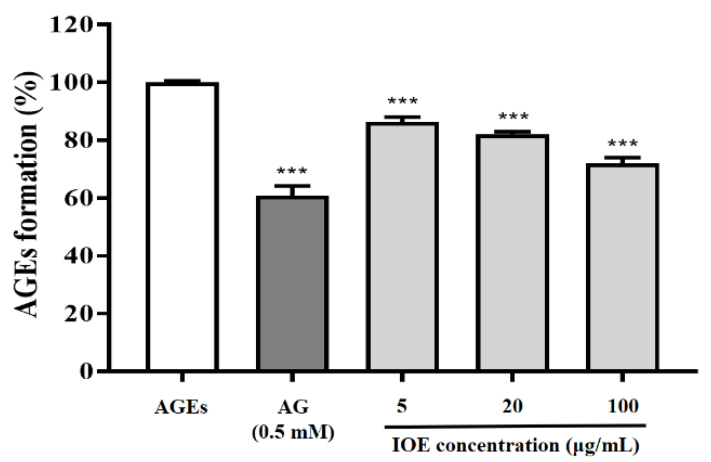

(C)

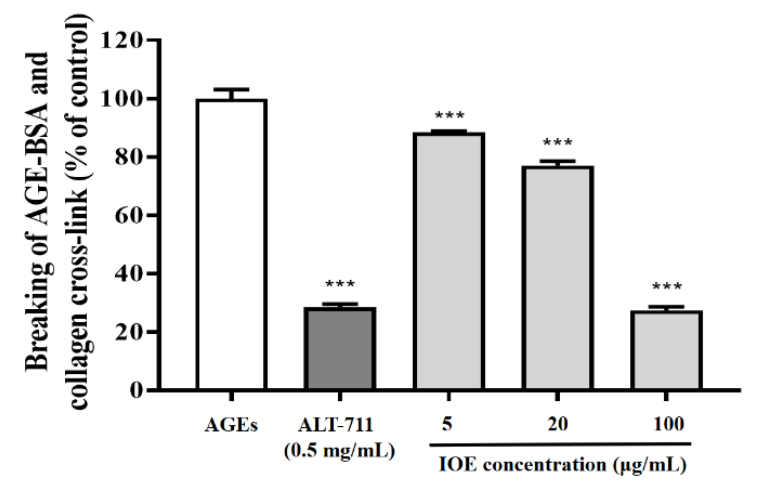

(B)

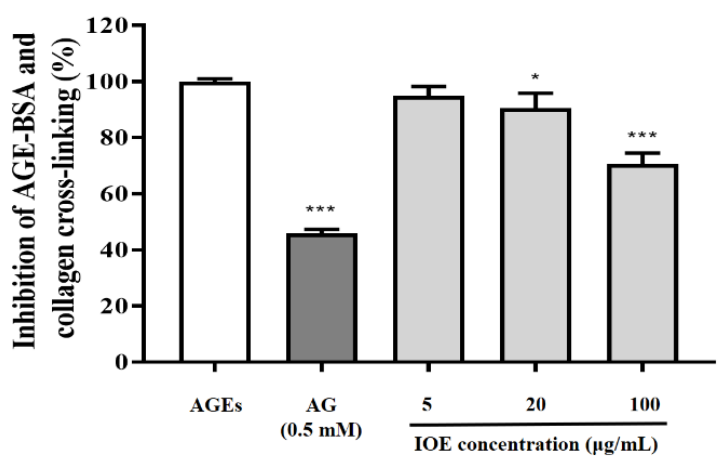

Figure 2. Effects of the Ishige okamurae extract on AGE-induced glycation reaction in vitro. Inhibition of AGE formation (A), inhibition of AGEs-BSA and collagen cross-link formation (B), and breaking of AGEs-BSA and collagen cross-links (C). Bar values are expressed as mean \pm standard deviation $(n=6)\left(* p<0.05\right.$ and ${ }^{* * *} p<0.001$ vs. AGEs). 
ELISA was performed to evaluate the inhibitory ability of IOE on AGE-BSA and collagen cross-linking. The AGE-BSA mixture considerably increased cross-linking between the AGEs and collagen, but treatment with IOE significantly $\left(* p<0.05\right.$ or $\left.{ }^{* * *} p<0.001\right)$ inhibited the formation of cross-links between the AGEs and collagen in a dose-dependent manner (Figure 2B). In particular, IOE treatment, at a concentration of $100 \mu \mathrm{g} / \mathrm{mL}$ displayed a cross-linking inhibitory effect identical to that of AG, which was used as a positive control.

The effect of IOE on breaking the cross-links formed between AGEs and collagen was evaluated using ELISA (Figure 2C). IOE treatment was confirmed to effectively cleave the cross-links formed between AGEs and collagen, at a level comparable to that of ALT-711, which is known to serve as a representative cross-link breaker. Overall, upon evaluating the anti-glycation property of IOE through various in vitro models, it was confirmed that IOE treatment not only inhibited AGE formation, but also inhibited the formation of, and destroyed the cross-links formed between, AGEs and collagen.

\subsection{Protective Effects of I. okamurae against MGO-Induced Renal Damage in Mesangial Cells}

I. okamurae has strong antioxidant effects in vitro. Thus, we evaluated whether IOE can relieve oxidative stress caused upon MGO exposure. First, we examined the cytotoxicity of IOE using an MTT assay. A decrease in cell viability to lower than $80 \%$ compared to the non-treated group was considered cytotoxic. All the concentrations $(5,20$, and $100 \mu \mathrm{g} / \mathrm{mL})$ of IOE tested in this study were found to be non-cytotoxic to mesangial cells (data not shown). MGO, which is one of the major contributory elements of diabetes complications, can induce renal damage in various biological and pathological processes, resulting in cell death. The cell viability of mesangial cells exposed to MGO was lower (approximately $67 \%$ decrease) than control cells (non-treated group) (100\%) (Figure 3A). Treatment with IOE significantly (\# $p<0.05$ or \#\#\# $p<0.001$ ) increased cell viability, as compared to the MGO-treated group, in a dose-dependent manner. Pre-treatment with $100 \mu \mathrm{g} / \mathrm{mL}$ of IOE increased mesangial cell viability by up to approximately $55 \%$, and exhibited the same level of nephroprotective effects as $0.5 \mathrm{mM} \mathrm{AG}$, which was used as a positive control. Thus, it was confirmed that IOE protected mouse glomerular mesangial cells from MGOinduced oxidative stress at all the treatment concentrations, which may help treat diabetic complications such as nephropathy.

The intracellular antioxidant capacity of IOE was confirmed by performing a DCFHDA assay to confirm the ability of IOE to reduce MGO-induced intracellular ROS production in mouse glomerular mesangial cells (Figure 3B). When mesangial cells were exposed to $0.5 \mathrm{mM}$ MGO (used as oxidative stress) for $1 \mathrm{~h}$, there was a significant (*** $p<0.001$ ) increase in the ROS production levels, up to approximately $427 \%$, as compared to the non-treated control (100\%). AG (0.5 mM; used as positive control) reduced MGO-induced intracellular ROS production level to approximately $147 \%$. Pre-treatment of mesangial cells with IOE caused a significant decrease in intracellular ROS production in a dosedependent manner (\# $p<0.05$ or (\#\#\# $p<0.001$ ). The intracellular antioxidant capacity of $100 \mu \mathrm{g} / \mathrm{mL}$ IOE suppressed ROS production by up to $199 \%$. The results of this in vitro study showed that pretreatment with IOE had a remarkable renoprotective effect, by inhibiting MGO-induced oxidative stress.

MGO treatment is known to increase intracellular MGO accumulation and MGOderived AGE concentration, resulting in MGO-induced oxidative cell damage and cell apoptosis in diverse cell lines. In this study, it was confirmed that MGO treatment significantly increased MGO accumulation in mesangial cells, while IOE treatment significantly reduced intracellular MGO-protein adduct concentration (\#\# $p<0.001$ ) (Figure 3C). 
(A)

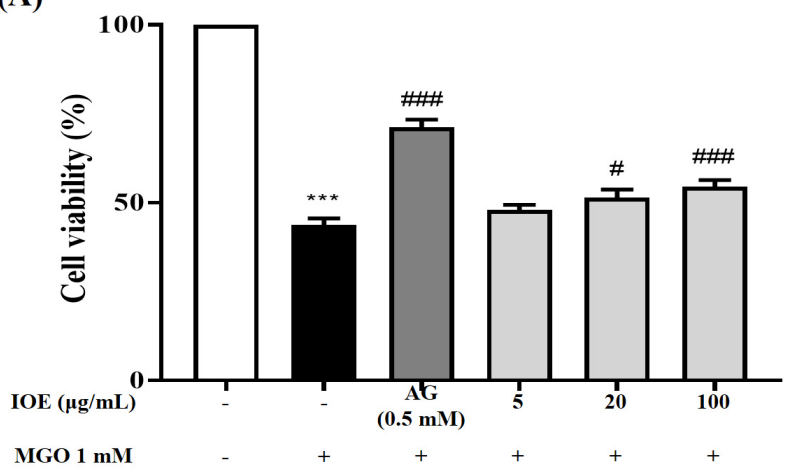

(C)

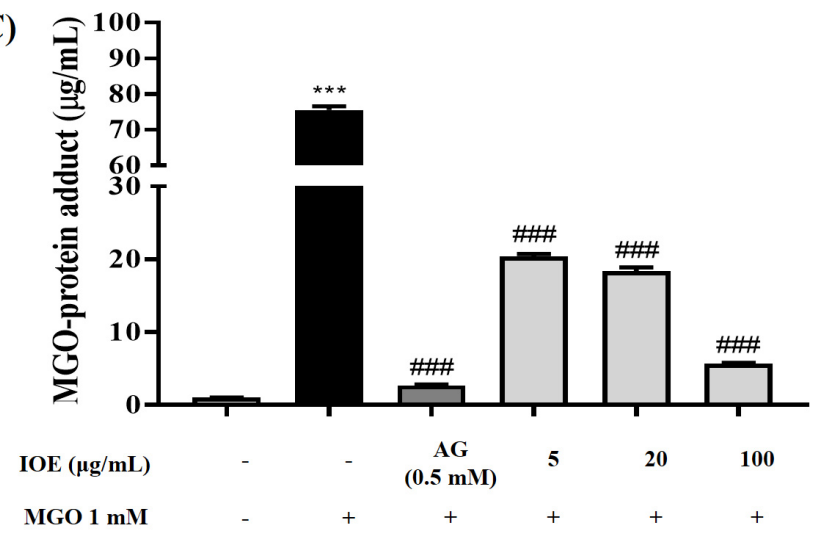

(B)

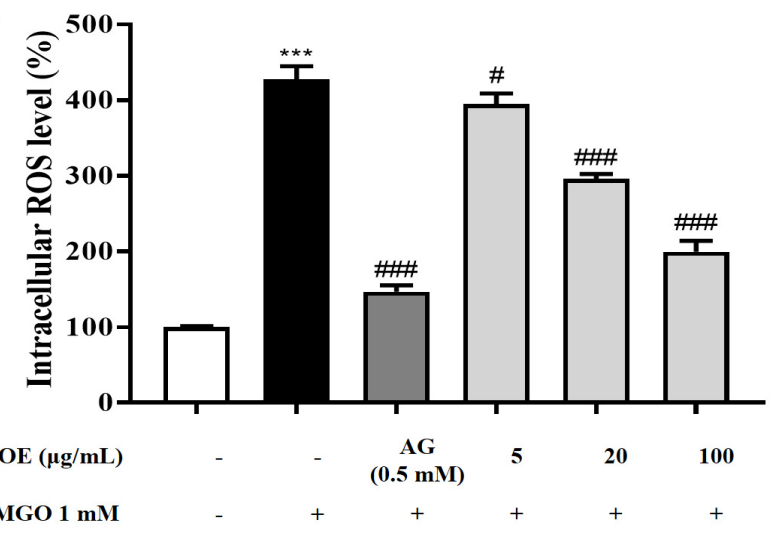

Figure 3. Protective effects of IOE on MGO-induced renal damage. Protective effect (A), intracellular antioxidant capacity (B) and MGO-cross-linked protein accumulation inhibitory effect (C)of IOE on MGO-induced renal damage in mouse glomerular mesangial cells. A decrease in cell viability to lower than $80 \%$ compared to non-treated group was considered to be cytotoxic. Each experiment was performed three times independently. Bar values are presented as mean \pm standard deviation ( $n=6$ ). ( ${ }^{* * *} p<0.001$ vs. normal group and $\# p<0.05$, \#\#\# $p<0.001$ vs. MGO-treated group).

\subsection{Preventive Effects of I. okamurae against MGO-Induced Apoptotic Cell Death}

The apoptotic cascade of cells is known to induce the cleavage of protein substrates and apoptotic cell death. Quantitative measurement of apoptotic cell death was carried out using flow cytometry with the Muse ${ }^{\mathrm{TM}}$ Annexin V \& Dead Cell Kit. Apoptotic cells were stained with annexin V (FITC), whereas necrotic cells were stained with PI (Figure 4A). Treatment of mesangial cells with MGO significantly $\left({ }^{* * *} p<0.001\right)$ reduced the percentage of live cells from $90.0 \%$ to $25.6 \%$ and increased the percentage of total apoptotic cells from $9.4 \%$ to $71.1 \%$. However, when mesangial cells were treated with IOE, apoptotic cell death was significantly suppressed (\#\#\# $p<0.001$ ), up to $21.2 \%$ at an IOE concentration of $100 \mu \mathrm{g} / \mathrm{mL}$ (Figure 4B). In addition, it was confirmed that IOE treatment suppressed the MGO-induced apoptotic cascade of mesangial cells, thereby maintaining a level of live cells similar to that seen in the normal group (Figure 4B). These results showed that IOE has the potential to prevent AGE-related renal damage by effectively inhibiting MGO-induced cell death, intracellular ROS production, intracellular MGO-protein adduct accumulation, and apoptotic cell death. 

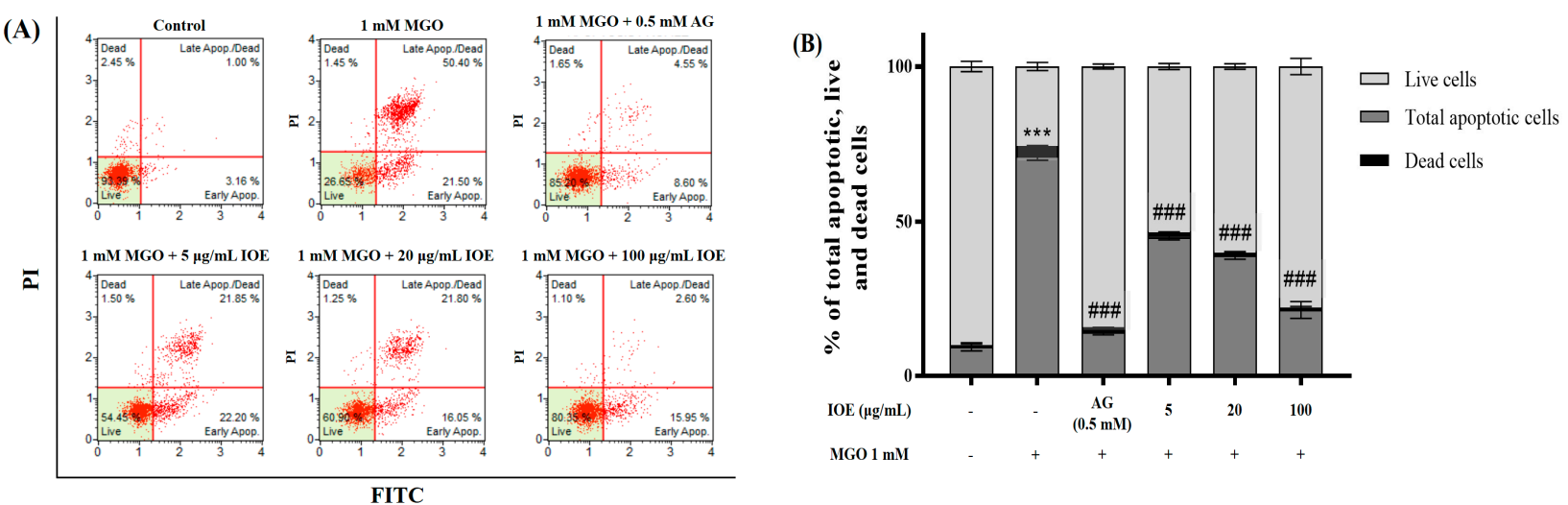

Figure 4. Protective effects of IOE on MGO-induced apoptotic cell death. Preventive effect of IOE on MGO-induced apoptosis in mouse glomerular mesangial cells. Dot plots for flow cytometric analysis of apoptotic cells. Annexin $\mathrm{V}-$ positive cells were assigned to the upper-right (late stage apoptotic cells) and lower-right (early stage apoptotic cells) quadrants, respectively. Dead cells are presented in the upper-left in the dot plots. Living cells without signs of apoptosis in the lower-left quadrant were negative for both Annexin V \& PI staining (A). Bar graph showing the percentage of live, apoptotic (early and late apoptotic), and dead cells determined using the Muse ${ }^{\mathrm{TM}}$ Annexin V \& Dead Cell Kit (B). Each experiment was performed three times independently. Bar values are presented as mean \pm standard deviation $(n=3)$ (*** $p<0.001$ vs. normal group and \#\#\# $p<0.001$ vs. MGO-treated group).

\subsection{Effects of I. okamurae on RAGE Expression in Mesangial Cells}

The increased formation of AGEs or accumulation of AGEs is a major contributor in the development of diabetic complications such as nephropathy. In addition, AGEs such as MGO are known to be directly involved in ROS production as well as transducing intracellular signals through RAGEs. Therefore, the effect of IOE on RAGE protein expression was evaluated using Western blots in mesangial cells (Figure 5A). MGO treatment increased RAGE protein expression by approximately 4-fold, as compared to normal cells (non-treated group). On the other hand, IOE treatment significantly (\#\#\# $p<0.001$ ) suppressed MGO-induced RAGE protein expression (Figure 5B). In addition, IOE not only effectively suppressed RAGE protein expression at all the tested concentrations, but also exhibited an inhibitory effect on RAGE protein expression that corresponded with that of AG, which was used as a positive control.

\subsection{Effects of I. okamurae on Nrf2/ARE Pathway}

To assess the preventive ability of IOE against MGO-induced renal damage, the Nrf2/ARE signaling pathway was investigated by Western blotting (Figure 6A). Nrf2 protein expression was significantly lower in MGO-treated cells compared to the control group (Figure 6B). The expression of Nrf2 protein was significantly increased in $2 \mu \mathrm{g} / \mathrm{mL}$ IOE-treated cells and was similar to that of AG-treated cells. Nrf2 protein expression increased in a concentration-dependent manner. Furthermore, MGO treatment significantly reduced the expression of antioxidant enzymes such as HO-1, NQO1, CAT, and SOD1 in mesangial cells (*** $p<0.001$ ), whereas IOE treatment significantly improved (\#\#\# $p<0.001$ ) (Figure 6C-F). These results indicate that IOE can protect mesangial cells from MGO-induced oxidative stress by increasing the expression of proteins involved in the Nrf2/ARE pathway. 
(A)

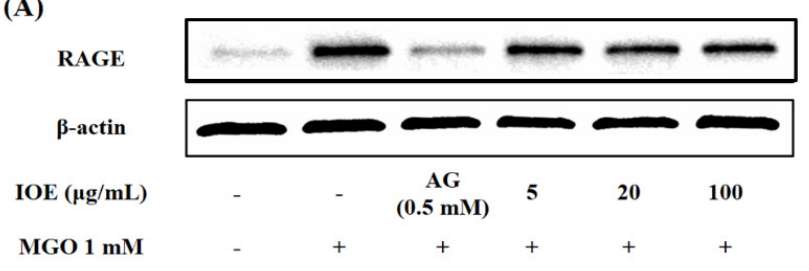

(B)

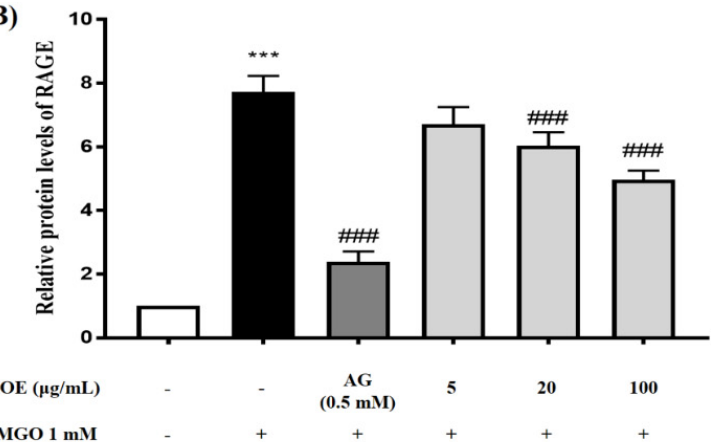

Figure 5. Effects of IOE on RAGE protein expression in MGO-induced mouse glomerular mesangial cells. Cells were pretreated with IOE for $1 \mathrm{~h}$, followed by incubation with $1 \mathrm{mM}$ MGO for $24 \mathrm{~h}$. The protein expression levels of RAGE were measured using Western blots (A). RAGE band intensity; $\beta$-actin was used as an internal control (B). Each experiment was performed three times independently. Bar values are presented as mean \pm standard deviation $(n=3)\left({ }^{* * *} p<0.001 \mathrm{vs}\right.$. normal group and \#\#\# $p<0.001$ vs. MGO-treated group).

(A)

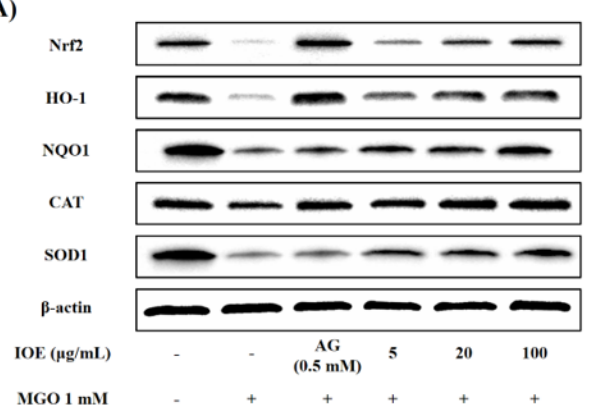

(C)

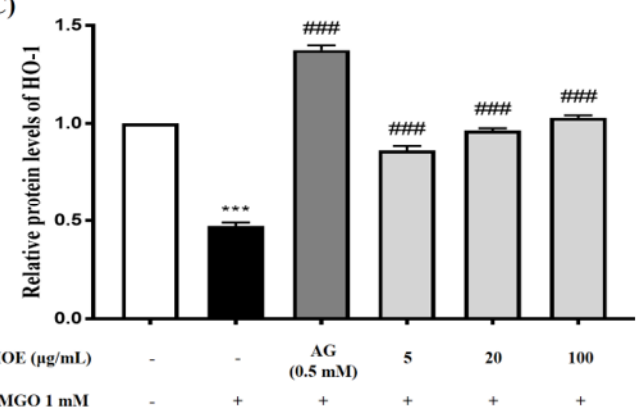

(E)

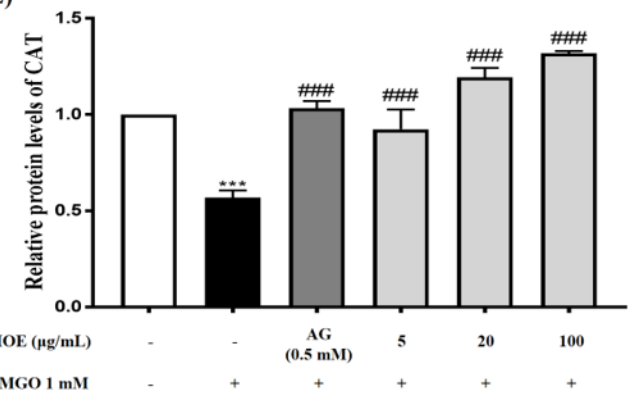

(B)

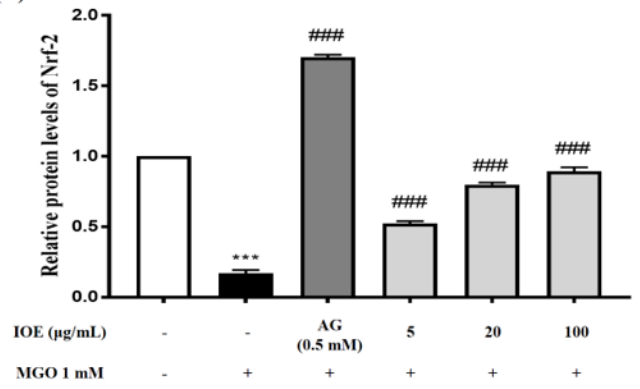

(D)

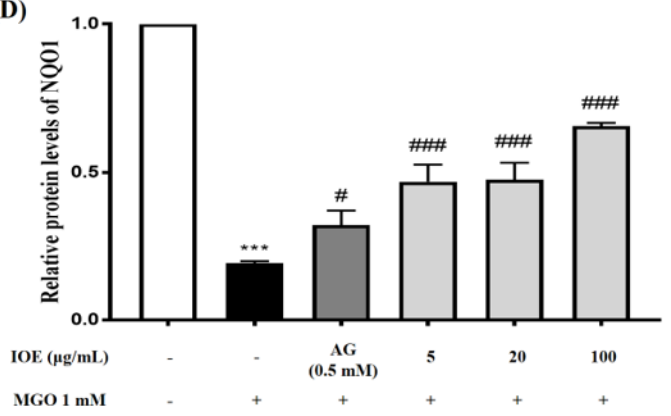

(F)

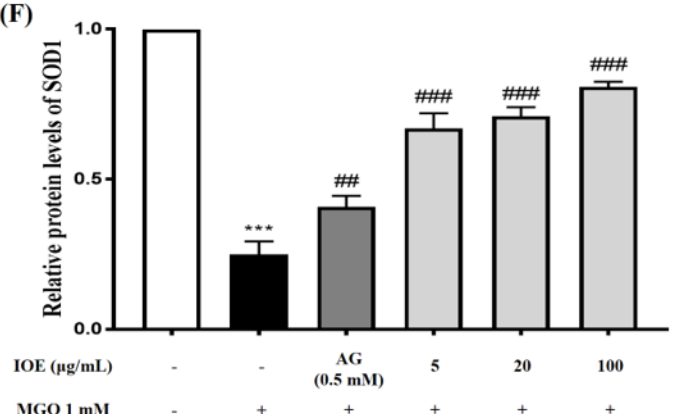

Figure 6. Effect of IOE on expression of Nrf2/ARE in MGO-induced mouse glomerular mesangial cells. Cells were pretreated with IOE for $1 \mathrm{~h}$, followed by incubation with $1 \mathrm{mM} \mathrm{MGO}$ for $24 \mathrm{~h}$. The protein expression levels of Nrf2, HO-1, NQO1, CAT, and SOD1 were measured using Western blot (A). Nrf2, HO-1, NQO1, CAT, and SOD1 band intensity; $\beta$-actin was used as an internal control (B-F). Each experiment was performed three times independently. Bar values are presented as mean \pm standard deviation $(n=3)\left(^{* * *} p<0.001\right.$ vs. normal group and $\# p<0.05, \# \#<0.01$, and \#\#\# $p<0.001$ vs. MGO-treated group). 


\subsection{Effects of I. okamurae on MAPK Phosphorylation}

Phosphorylation-induced activation of MAPK is a crucial stage in MGO-induced apoptosis. As shown in Figure 7A, the phosphorylation of ERK, JNK, and p38 in MGOtreated mesangial cells was analyzed by Western blotting using the following antibodies: p-ERK, ERK, p-JNK, JNK, p-p38, and p38. Treatment of mesangial cells with MGO caused a significant increase in the phosphorylation of ERK, JNK, and p38. Conversely, IOE pretreatment suppressed their phosphorylation (Figure 7B-D).
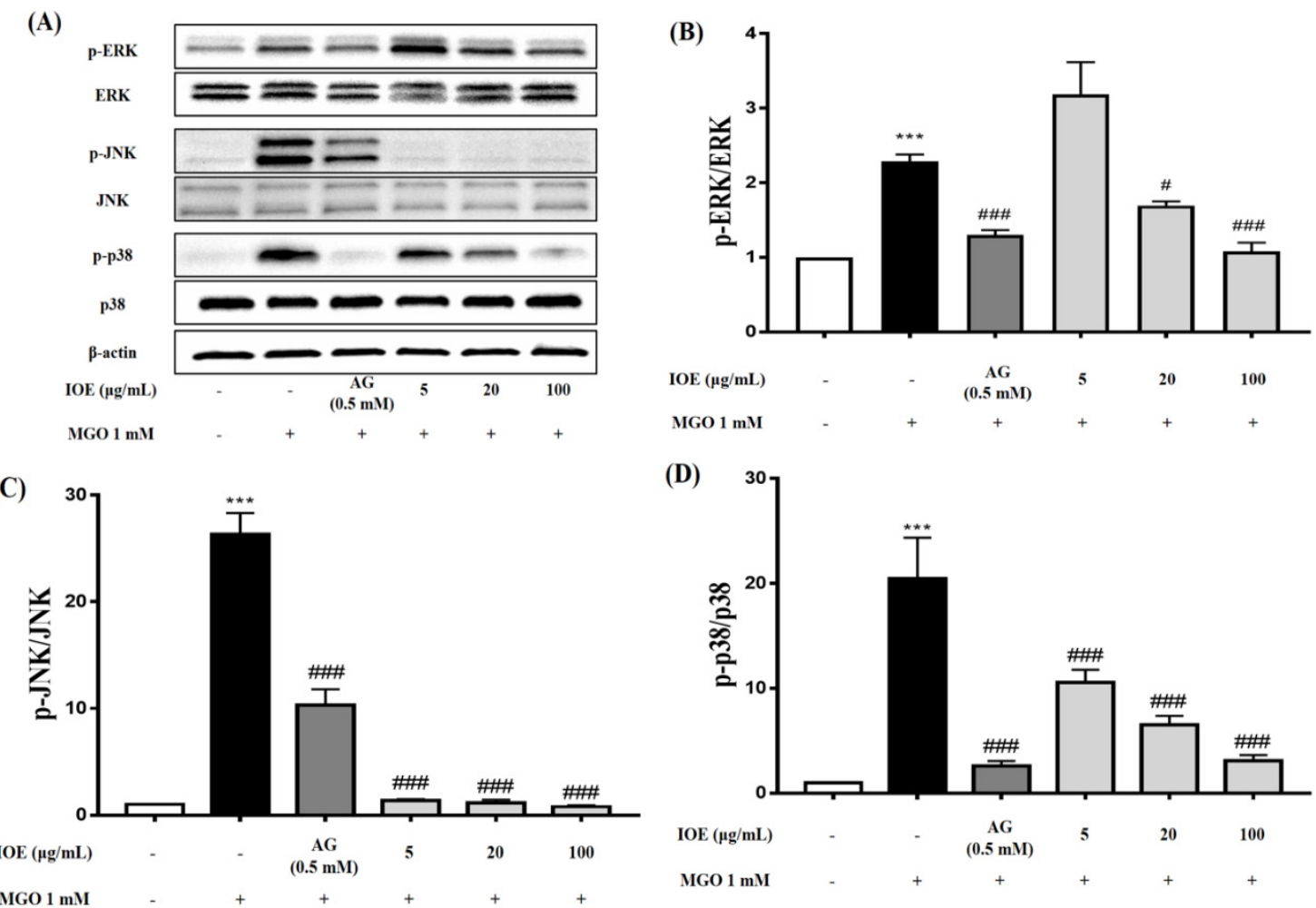

Figure 7. Effect of IOE on MAPK signaling pathways in MGO-induced mouse glomerular mesangial cells. Cells were pretreated with IOE for $1 \mathrm{~h}$, followed by incubation with $1 \mathrm{mM} \mathrm{MGO}$ for $24 \mathrm{~h}$. The protein expression levels of MAPKs (p-ERK, ERK, p-JNK, JNK, p-p38, and p38) were examined using Western blot (A). p-ERK/ERK, p-JNK/JNK, and $\mathrm{p}-\mathrm{p} 38 / \mathrm{p} 38$ ratios $(\mathbf{B}-\mathbf{D})$. Data are presented as mean \pm standard deviation $(n=3){ }^{* * *} p<0.001$ vs. normal group and $\# p<0.05$, and \#\#\# $p<0.001$ vs. MGO-treated group).

\section{Discussion}

Under hyperglycemic conditions, the carbonyl group of sugar and the free amino group of biological proteins become AGEs. During glycation, reducing sugars are dicarbonyl compounds, including MGO, glyoxal, and 3-deoxyclucosone. Of these, MGO is the most reactive compound, and well known for its glycation potency [7]. MGO has been shown to induce aging, retinopathy, cardiovascular diseases, and renal dysfunction via dicarbonyl stress in vivo [6]. In addition, elevated levels of MGO in Drosophila have been shown to induce fatty acid synthase, hyperglycemia, MGO-adducts, and insulin resistance [21]. Moreover, accumulation of AGEs such as MGO in the tissues is implicated in AGE-related diabetes complications because it alters enzyme activity, reduces ligand binding, modifies protein half-life, and alters immunogenicity [22]. Therefore, inhibiting the accumulation of AGEs, suppressing the production of AGEs, inhibiting/breaking crosslinks between AGEs and proteins, or reducing AGE-induced oxidative stress is believed to be an effective strategy to prevent and delay AGE-related diabetic complications.

I. okamurae, an edible brown alga, is known to be effective in ameliorating blood glucose levels and insulin resistance, as well as, exhibiting strong antioxidant activity [13,23]. Several previous studies have reported that natural plant resources, especially those with 
potent antioxidant compounds appear to play a vital action in the improvement of disorders involving oxidative stress-related diabetes mellitus [24]. Additionally, DPHC and IPA, which are representative compounds derived from I. okamurae, are known to suppress elevated blood glucose levels after a meal and improve glucose homeostasis $[13,15]$. Therefore, we determined the DPHC and IPA contents of I. okamurae using HPLC analysis (Figure 1). In our study, IOE showed anti-glycation properties by inhibiting AGE formation and breaking/inhibiting AGEs-collagen cross-links (Figure 2). These results indicated that I. okamurae inhibits AGEs through a variety of mechanisms, suggesting that it is effective in preventing and delaying AGE-related diabetes complications.

Chronic hyperglycemic conditions further accelerate this reaction and increase the accumulation of AGEs in body tissues. AGE accumulation has been implicated in the development of insulin resistance and AGE-related diabetic complications. MGO treatment increases intracellular MGO concentrations and MGO-derived AGE concentrations, subsequently causing cellular damage and apoptotic cell death by oxidative stress in diverse cells [25]. In addition, MGO, which is a reactive dicarbonyl compound of AGEs, increases intracellular oxidative stress and induces apoptosis [7]. In the present study, I. okamurae treatment exhibited protective effects against MGO-induced renal damage and intracellular ROS accumulation in mouse kidney mesangial cells (Figure 3). In addition, I. okamurae not only significantly reduced intracellular MGO accumulation, but also inhibited apoptotic cell death mediated by MGO-induced oxidative stress (Figure 4). Therefore, our results demonstrated that IOE protects against MGO-induced cytotoxicity by downregulating MGO-induced ROS generation, MGO-cross-linked protein concentration, and apoptotic cell death.

Our results indicated that IOE significantly reduced the level of apoptosis caused by MGO, but the mechanism of action was not clear. We hypothesized that certain signaling pathways activated by MGO may be involved in the mechanism of action. To determine whether RAGE plays an important role in MGO-induced cell dysfunction, we first measured the protein expression of the AGE receptor RAGE (Figure 5). RAGE expression increased upon MGO treatment, but significantly decreased upon IOE treatment. The interaction of AGEs with RAGE enhances oxidative stress through ROS production by NADPH oxidases inside the mitochondria [26].

We hypothesized that IOE would suppress MGO-induced toxicity by causing a change in the Nrf2 signaling pathway while reducing RAGE expression. Nrf2 is an essential regulator of ARE-mediated induction, including the regulation of antioxidant enzymes such as SOD, NQO1, and catalase. Several studies have reported that Nrf2 regulates AGEinduced inflammatory cytokine concentrations and oxidative stress [27]. The transcription factor Nrf2 normally forms a complex with the cytoskeleton-associated protein Keap1 in the cytoplasm. When cells are exposed to oxidative stress, the Nrf2/Keap1 interaction is broken and free Nrf2 translocates into the nucleus to activate ARE-dependent genes [28,29]. To determine whether IOE can activate Nrf2 under MGO treatment, we performed a Western blot with MGO-treated samples. In our study, MGO treatment significantly reduced Nrf2 protein expression and its downstream pathway molecules (Figure 6). However, IOE treatment significantly increased Nrf2 protein expression and the expression of antioxidant enzymes such as HO-1, NQO1, CAT, and SOD1. Therefore, our results suggest that IOE has an inhibitory effect on MGO-induced cytotoxicity by regulating the Nrf2 signaling pathway and reducing ROS production in renal cells.

MAPKs play an important role in cell differentiation and apoptosis. ERK, JNK, and p38 are major proteins in the MAPK group. ERK is associated with the proliferation and progression of certain cellular systems, but JNK, p38, and ERK are also associated with apoptosis [30,31]. In this study, we observed that pretreatment with IOE most dramatically inhibited the activation of ERK, JNK, and p38 (Figure 7). Inhibition of apoptosis by IOE was accompanied by inhibition of MAPK activation, suggesting that IOE can regulate the MAPK signaling pathway in MGO-treated intermediate cells. MAPKs can be activated independently and are involved in cell death. In recent years, it has been suggested that 
MGO-induced cytotoxicity is associated with the activation of members of the MAPK family, including ERK, JNK, and p38 [25]. Therefore, our results suggest that IOE has an inhibitory effect on MGO-induced cytotoxicity by regulating the Nrf2/ARE pathway and reducing ROS generation in renal cells.

An increase in the levels or accumulation of intracellular AGEs has been reported as one of the immediate causes of diabetes development and its related complications. Therefore, many researchers have attempted to prevent/improve/treat AGE-related diabetes and complications using natural resources or active compounds derived from them, as these present a low risk of side effects. Our results showed that I. okamurae, an edible brown alga, effectively inhibited various processes related to the production and accumulation of AGEs through actions such as inhibition of AGE formation, inhibition/breaking of AGE-protein cross-linking. In addition, it was demonstrated that I. okamurae exerts nephroprotective effects by reducing MGO-induced ROS generation and regulating the expression of proteins involved in the RAGE and Nrf2/ARE pathways in mesangial cells. Therefore, I. okamurae is considered a very useful natural resource with the potential to prevent and treat diabetic complications such as AGE-related diabetic nephropathy. However, since various physiologically active compounds exist in I. okamurae, additional studies using single phlorotannin compounds contained in I. okamurae are needed to clarify its efficacy. In addition, in order to verify the efficacy of the phlorotannin compound contained in $I$. okamurae for AGE-induced nephropathy, an in vivo experiment using an animal model is necessary.

\section{Conclusions}

In conclusion, our results clearly indicated that I. okamurae possesses anti-glycation activity. In addition, it was demonstrated that I. okamurae effectively protects renal cells by reducing MGO-induced ROS generation and oxidative stress, as well as increasing the expression of proteins involved in antioxidant defense mechanisms. Consequently, I. okamurae may be used as a potential natural therapeutic agent for the management of AGE-related diabetic complications as an AGE inhibitor and breaker. In particular, I. okamurae containing phlorotannins such as IPA and DPHC should be considered to be effective in preventing AGE-related nephropathy.

Author Contributions: Conceptualization, C.C. and S.-H.L.; methodology, C.C., C.L. and M.K.; software, C.C., C.L. and M.K.; validation, M.K. and S.K.; formal analysis, C.C., C.L., and M.K.; investigation, C.C., C.L., M.K., B.R. and S.K.; resources, J.H. and S.-H.L.; data curation, C.C., M.K. and B.R.; writing-original draft preparation, C.C., C.L. and M.K.; writing-review and editing, J.H. and S.-H.L.; visualization, C.C., and C.L.; supervision, S.-H.L.; project administration, S.-H.L.; funding acquisition, S.-H.L. All authors have read and agreed to the published version of the manuscript.

Funding: This research was supported by a National Research Foundation of Korea (NRF) grant funded by the Korean government (Ministry of Science and ICT, MSIT) (No. NRF-2020R1A2C2012608) and a part of the project titled 'Development of seafood products for export diversification in response to strengthening sanitary inspection in japan', funded by the Ministry of Oceans and Fisheries, Korea.

Institutional Review Board Statement: Not applicable.

Informed Consent Statement: Not applicable.

Conflicts of Interest: The authors declare no conflict of interest. 


$\begin{array}{ll}\text { Abbreviations } \\ \text { IOE } & \begin{array}{l}\text { Ishige okamuare extract } \\ \text { advanced glycation end-products }\end{array} \\ \text { AGEs } & \begin{array}{l}\text { receptor for advanced glycation end-products } \\ \text { RAGE }\end{array} \\ \text { MGO } & \begin{array}{l}\text { methylglyoxal } \\ \text { diphlorethohydroxycarmalol }\end{array} \\ \text { DPHC } & \text { ishophloroglucin A } \\ \text { IPA } & \text { antioxidant response elements } \\ \text { ARE } & \text { reactive oxygen species } \\ \text { ROS } & \text { aminoguanidine } \\ \text { AG } & \text { alagebrium } \\ \text { ALT-711 } & \text { bovine serum albumin } \\ \text { BSA } & \text { 2,7-dichlorodi-hydrofluorescein diacetate } \\ \text { DCFH-DA } & \text { tetramethylbenzidine } \\ \text { TMB } & \text { phosphate buffered saline with Tween }{ }^{\circledR} 20 \\ \text { PBST } & \text { catalase } \\ \text { CAT } & \text { extracellular signal-regulated kinase } \\ \text { ERK } & \text { c-Jun N-terminal kinase } \\ \text { JNK } & \text { heme oxygenase-1 } \\ \text { HO-1 } & \text { NAD(P)H Quinone Oxidoreductase 1 } \\ \text { NQO1 } & \text { nuclear factor erythroid 2-related factor 2 } \\ \text { Nrf2 } & \text { superoxide dismutase } \\ \text { SOD1 } & \end{array}$

\section{References}

1. Huebschmann, A.G.; Regensteiner, J.G.; Vlassara, H.; Reusch, J.E.B. Diabetes and advanced glyxocidation end products. Diabetes Care 2006, 29, 1420-1432. [CrossRef]

2. Rowan, S.; Bejarano, E.; Taylor, A. Mechanistic targeting of advanced glycation end-products in age-related disease. Biochim. Ciophys. Acta Mol. Basis Dis. 2018, 1864, 3631-3643. [CrossRef] [PubMed]

3. Rabbani, N.; Thornalley, P.J. Advanced glycation end products in the pathogenesis of chronic kidney disease. Kidney Int. 2018, 93, 803-813. [CrossRef]

4. Yamagichi, S.I.; Nakamura, K.; Matsui, T. Regulration of advanced glycation end product (AGE)-receptor (RAGE) system by PPAR-gamma agonists and its implication in cardiovascular disease. Pharmacol. Res. 2009, 60, 174-178. [CrossRef] [PubMed]

5. Ahmed, N. Advanced glycation endproducts-role in pathology of diabetic complications. Diabetes Res. Clin. Pract. 2005, 67, 3-21. [CrossRef]

6. $\quad$ Brings, S.; Fleming, T.; Freichel, M.; Muckenthaler, M.U.; Herzing, S.; Nawroth, P.P. Dicarbonyls and advanced glycation end-products in the development of diabetic complications and targets for intervention. Int. J. Mol. Sci. 2017, 18, 984. [CrossRef] [PubMed]

7. Schalkwijk, C.G.; Stehouwer, C.D.A. Methylglyoxal, a highly reactive dicarbonyl compound, in diabetes, its vascular complications, and other age-related diseases. Physiol. Rev. 2020, 100, 407-461. [CrossRef]

8. Edelstein, D.; Brownlee, M. Mechanistic studies of advanced glycosylation end product inhibition by aminoguanidine. Diabetes 1992, 41, 26-29. [CrossRef] [PubMed]

9. Thornalley, P.J. Use of aminoguanidine (Pimagedine) to prevent the formation of advanced glycation endproducts. Arch. Biochem. Biophys. 2003, 419, 31-40. [CrossRef] [PubMed]

10. Heo, S.-J.; Jeon, Y.-J. Evaluation of diphlorethohydroxycarmalol isolated from Ishige okamurae for radical scavenging activity and its protective effect against $\mathrm{H}_{2} \mathrm{O}_{2}$-induced cell damage. Process Biochem. 2009, 44, 412-418. [CrossRef]

11. Kim, H.-S.; Wang, L.; Jayawardena, T.U.; Kim, E.-A.; Heo, S.-J.; Fernando, I.P.S.; Lee, J.-H.; Jeon, Y.-J. High-performance centrifugal partition chromatography (HPCPC) for efficient isolation of diphlorethohydroxycarmalol (DPHC) and screening of its antioxidant activity in a zebrafish model. Process Biochem. 2020, 88, 189-196. [CrossRef]

12. Kim, M.-M.; Rajapakse, N.; Kim, S.-K. Anti-inflammatory effect of Ishige okamurae ethanolic extract via inhibition of NF-кB transcription factor in RAW 264.7 cells. Phytother. Res. 2011, 93, 70-76.

13. Min, K.-H.; Kim, H.-J.; Jeon, Y.-J.; Han, J.-S. Ishige okamurae ameliorates hyperglycemia and insulin resistance in C57BL/KsJ-db/db mice. Diabetes Res. Clin. Pract. 2011, 93, 70-76. [CrossRef]

14. Yang, H.-W.; Son, M.; Choi, J.; Oh, S.; Jeon, Y.-J.; Byun, K. Effect of ishophloroglucin A, a component of Ishige okamurae, on glucose homeostasis in the pancreas and muscle of high fat diet-fed mice. Mar. Drugs 2011, 17, 608. [CrossRef] [PubMed]

15. Heo, S.-J.; Hwang, J.-Y.; Choi, J.-S.; Kim, H.-J.; Jeon, Y.-J. Diphlorethohydroxycarmalol isolated from Ishige okamurae, a brown algae, a potent $\alpha$-amylase inhibitor, alleviates postprandial hyperglycemia in diabetic mice. Eur. J. Pharmacol. 2009, 615, 252-256. [CrossRef] [PubMed] 
16. Do, M.H.; Choi, J.; Kim, Y.; Par, H.-Y.; Park, Y.; Ha, S.K. Schizonepeta tenuifolia reduces methylglyoxal-induced cytotoxicity and oxidative stress in mesangial cells. J. Funct. Foods. 2019, 62, 103531. [CrossRef]

17. Lee, J.-Y.; Oh, J.-G.; Kim, J.S.; Lee, K.-W. Effects of chebulic acid on advanced glycation endproducts-induced collagen cross-links. Biol. Pharm. Bull. 2014, 37, 1162-1167. [CrossRef] [PubMed]

18. Do, M.H.; Hur, J.Y.; Choi, J.W.; Kim, M.N.; Kim, M.J.; Kim, Y.S.; Ha, S.K. Eucommia ulmoides ameliorates glucotoxicity by suppressing advanced glycation end-products in diabetic mice kidney. Nutrients 2018, 10, 265. [CrossRef] [PubMed]

19. Ryu, B.; Jiang, Y.; Kim, H.-S.; Hyun, J.-M.; Lim, S.-B.; Li, Y. Ishophloroglucin A, a novel phlorotannin for standardizing the anti- $\alpha$-glucosidase activity of Ishige okamurae. Mar. Drugs 2018, 16, 375. [CrossRef]

20. Fernando, K.H.N.; Yang, H.-W.; Jiang, Y.; Jeon, Y.-J.; Ryu, B. Diphlorethohydroxycarmalol isolated from Ishige okamurae represses high glucose-induced angiogenesis in vitro and in vivo. Mar. Drugs 2018, 16, 375. [CrossRef]

21. Moraru, A.; Wiederstein, J.; Pfaff, D.; Fleming, T.; Miller, A.K.; Nawroth, P. Elevated levels of the reactive metabolite methylglyoxal recapitulate progression of type 2 diabetes. Cell Metab. 2018, 27, 926-934. [CrossRef]

22. Ahmed, N.; Thornalley, P.J. Advanced glycation endproducts: What is their relevance to diabetic complications? Diabetes Obes. Metab. 2006, 9, 233-245. [CrossRef]

23. Zou, Y.; Qian, Z.-J.; Li, Y.; Kim, M.-M.; Lee, S.-H.; Kim, S.-K. Antioxidant effects of phlorotannins isolated from Ishige okamurae in free radical mediated oxidative systems. J. Agric. Food Chem. 2008, 56, 7001-7009. [CrossRef] [PubMed]

24. Rahimi, R.; Nikfar, S.; Larijani, B.; Abdollahi, M. A review on the role of antioxidants in the management of diabetes and its complications. Biomed. Pharmacother. 2005, 59, 365-373. [CrossRef] [PubMed]

25. Liu, B.-F.; Miyata, S.; Hirota, Y.; Higo, S.; Miyazaki, H.; Fukunaga, M.; Hamada, Y.; Ueyama, S.; Muramoto, O.; Uriuhara, A.; et al. Methylglyoxal induces apoptosis through activation of p38 mitogen-activated protein kinase in rat mesangial cells. Kidney Int. 2003, 63, 947-957. [CrossRef] [PubMed]

26. Cepas, V.; Colino, M.; Mayo, J.C.; Sainz, R.M. Redox signaling and advanced glycation endproducts (AGEs) in diet-related disease. Antioxidants 2020, 9, 142. [CrossRef]

27. Li, S.; Song, Z.; Liu, T.; Liang, J.; Yuan, J.; Xu, Z. Polysaccharide from Ostrea rivularis attenuates reproductive oxidative stress damage via activating keap1-Nrf2/ARE pathway. Carbohydr. Polym. 2018, 186, 321-331. [CrossRef]

28. Kim, S.K.; Yang, J.W.; Kim, M.R.; Roh, S.H.; Kim, H.G.; Lee, K.Y. Increased expression of Nrf2/ARE-dependent anti-oxidatn proteins in tamoxifen-resistant breast cancer cells. Free Radic. Biol. Med. 2008, 45, 537-546. [CrossRef]

29. Kobayashi, A.; Ohta, T.; Yamamoto, M. Unique function of the Nrf2-Keap1 pathway in the inducible expression of antioxidant and detoxifying enzymes. Methods Enzymol. 2004, 378, 273-286.

30. Choi, A.Y.; Choi, J.H.; Yoon, H.N.; Hwang, K.-Y.; Noh, M.H.; Choe, W.C.; Yoon, K.-S.; Ha, J.H.; Yeo, E.-J.; Kang, I.S. Luteolin induces apoptosis through endoplasmic reticulum stress and mitochondrial dysfunction in neuro-2a mouse neuroblastoma cells. Eur. J. Pharmacol. 2011, 668, 115-126. [CrossRef]

31. Arai, K.; Lee, S.-R.; Leyen, K.V.; Kurose, H.; Lo, E.H. Involvement of ERK MAP kinase in endoplasmic reticulum stress in SH-SY5Y human neuroblastoma cells. J. Neurochem. 2004, 89, 232-239. [CrossRef] [PubMed] 